\title{
Characterization and Morphological Analysis of Pearlite in a Eutectoid Steel
}

\author{
F. G. Caballero ${ }^{1,2}$, C. García de Andrés ${ }^{1}$ and C. Capdevila ${ }^{1,2}$ \\ 1 Department of Physical Metallurgy, Centro Nacional de Investigaciones \\ Metalúrgicas (CENIM), Consejo Superior de Investigaciones Científicas (CSIC), \\ Avda. Gregorio del Amo, 8, 28040 Madrid, Spain and ${ }^{2}$ Department of Materials \\ Science and Metallurgy, University of Cambridge, Pembroke Street, Cambridge CB2 \\ 3QZ, UK
}

Abbreviated Title: Characterization of Pearlite in Steels

\begin{abstract}
Three different morphologies of pearlite have been formed isothermally at three temperatures in a eutectoid steel. The interlamellar spacings of these morphologies have been measured by scanning and transmission electron microscopy. A comparison of these experimental results and the interlamellar spacing calculations using a theoretical model previously developed has allowed some standardisation to be established for the morphological characterisation of pearlite, in terms of sample preparation, examination conditions and quantitative metallography procedures. It has been found that using scanning electron microscopy the morphological characterisation of pearlite with interlamellar spacing finer than $0.085 \mu \mathrm{m}$ could lead to unreliable results, since the strain induced during metallographic preparation of very fine pearlite destroys the lamellar morphology of pearlite. In that case, it is
\end{abstract}


advisable to measure the interlamellar spacing of pearlite using transmission electron microscopy.

\section{INTRODUCTION}

Pearlite is a lamellar product of eutectoid decomposition, which may form in steels and non-ferrous alloys during transformations under isothermal or continuous-cooling conditions [1,2]. A pearlite nodule is composed of multiple colonies; each colony has parallel lamellae, which are orientated differently with respect to lamellae in adjacent colonies. This also exhibits a wide range of interlamellar spacings in different colonies because of intersection with the polishing plane of pearlite colonies at different angles. The interlamellar spacing is reflected by the diffusion kinetics at the transformation front and is a sensitive parameter that, in a particular steel, is larger the higher the transformation temperature [3]. Mehl and co-workers [3] demonstrated that the spacing decreased as the degree of undercooling, $\Delta T$, below the eutectoid temperature increased. Zener [4] provided the first theoretical analysis of these observations, which allows calculation of the interlamellar spacing of pearlite as a function of undercooling.

In the present work, three different morphologies of pearlite were isothermally formed at three temperatures in a eutectoid steel. The interlamellar spacings of these morphologies were measured by scanning and transmission electron microscopy. Moreover, the interlamellar spacings were calculated by means of a model previously published elsewhere [5]. This model was developed using the theoretical method proposed by Zener [4] and Takahashi empirical expression [6]. That allowed 
establishment of some standardisation for the morphological characterisation of pearlite, in terms of sample preparation, examination conditions and quantitative metallography procedure.

\section{EXPERIMENTAL PROCEDURE}

The chemical composition of the studied steel is presented in Table 1. Cylindrical samples of $2 \mathrm{~mm}$ in diameter and $12 \mathrm{~mm}$ in length were austenitised for 5 minutes at $1273 \mathrm{~K}$, isothermally transformed at temperatures ranging from 798 to $948 \mathrm{~K}$ for different times and subsequently cooled rapidly to room temperature. Table 2 lists all the temperatures and holding times used for the isothermal decomposition of austenite in this steel.

The heating and cooling devices of an Adamel Lhomargy DT1000 high-resolution dilatometer have been used to perform all the heat treatments previously mentioned. This dilatometer is equipped with a radiation furnace for heating. The power radiated by two tungsten filament lamps is focused on the specimen by means of a bi-elliptical reflector. The temperature is measured with a $0.1 \mathrm{~mm}$ diameter Chromel-Alumel (type K) thermocouple welded to the specimen. Cooling is carried out by blowing a jet of helium gas directly onto the specimen surface. The helium flow rate during cooling is controlled by a proportional servovalve. The excellent efficiency of heat transmission and the very low thermal inertia of the system ensure that the heating and cooling rates, ranging from $0.003 \mathrm{C} \%$ s to approximately $200 \mathrm{C} / \mathrm{s}$, remain constant.

Specimens were polished in the usual way and finished on $0.25 \mu \mathrm{m}$ diamond paste. It was found that long polishing times, particularly on the $6 \mu \mathrm{m}$ pad, resulted in a deep 
worked layer that produced a distorted lamellar structure. Cementite plates in final microstructure seem to bend and eventually fracture during plastic deformation of ferrite plates induced by long polishing periods [7] (see Fig. 1). Polishing times up to five minutes on the $0.25 \mu \mathrm{m}$ pad were found to be adequate to remove evidence of surface deformation caused by previous pads. Deep primary etching with a solution of picric acid in isopropyl alcohol with several drops of Vilella's reagent was used to ensure that any deformed layer introduced by polishing was removed. This etching was eliminated using the 1 and $0.25 \mu \mathrm{m}$ diamond pads with almost no pressure being exerted on the sample for no longer than 3 to 4 minutes. The sample was then etched again, this time lightly, and polished carefully on the 1 and $0.25 \mu \mathrm{m}$ diamond pad. Finally, a light etch was given to the sample.

This repeated polishing-etching procedure allows disclosure of the morphology of pearlite in specimens MORF1-3 on a JEOL JXA-820 scanning electron microscope (Figs. 2(a)-(c)). The morphology of pearlite was also revealed by transmission electron microscopy in all the specimens. With the aim of preparing specimens for transmission electron microscopy, 3 mm diameter cylindrical samples were sliced into $100 \mu \mathrm{m}$ thick discs and subsequently ground down to foils of $50 \mu \mathrm{m}$ thickness on wet 800 grit silicon carbide paper. These foils were finally electropolished until perforation occurred in a twin-jet electropolisher at $-5{ }^{\circ} \mathrm{C}$ and a voltage of $40 \mathrm{~V}$ using a solution of $5 \%$ perchloric acid, $15 \%$ glycerol and $80 \%$ methanol. The foils were examined in a JEOL-JEM 2010 transmission electron microscope at an operating voltage of $200 \mathrm{kV}$ (Figs. 3(a)-(c)).

The mean true interlamellar spacing $\left(\sigma_{o}\right)$ was derived from electron micrographs according to Underwood's intersection procedure. Underwood [8] recommends determining the mean random spacing $\sigma_{r}$ first to estimate the mean true spacing $\sigma_{o}$. 
For this purpose, a circular test grid of diameter $d_{c}$ is superimposed on an electron micrograph. The number $n$ of intersections of lamellae of carbide with the test grid is counted. This procedure is repeated on a number of fields chosen randomly. Then, the mean random spacing $\sigma_{r}$ is calculated from:

$$
\sigma_{r}=\frac{\pi d_{c}}{n M}
$$

where $M$ is the magnification of the micrograph.

Saltykov [9] has shown that, for pearlite with a constant spacing within each colony, the mean true spacing $\sigma_{o}$ is related to the mean random spacing $\sigma_{r}$ by:

$\sigma_{o}=0.5 \sigma_{r}$

Data for $\sigma_{o}$ are listed in Table 3.

\section{RESULTS AND DISCUSSION}

The formation of pearlite is a good example of reconstructive transformation and known to show a constant growth rate. The growth rate of pearlite is believed to be controlled by volume diffusion of carbon in austenite ahead of the interface $[4,10]$. The diffusion of carbon plays a more important role than that of substitutional alloying elements, since the diffusivity of the substitutional alloying elements in austenite is far smaller than that of carbon. As a result, the substitutional alloying 
elements may not diffuse a long distance during the reaction. The growth rate of pearlite, in that case, is expressed as follows [10]:

$$
G_{v}=K_{v} \frac{1}{\sigma_{o}}\left(1-\frac{\sigma_{c}}{\sigma_{o}}\right)
$$

where $G_{v}$ is the volume diffusion controlled growth rate of pearlite, $K_{v}$ is constant at a given temperature, $\sigma_{o}$ is the interlamellar spacing and $\sigma_{c}$ is the theoretical critical spacing at zero growth rate.

Since Eq. (3) contains two unknown parameters, $G_{v}$ and $\sigma_{o}$, for a given temperature $T$, it does not provide a unique solution. However, Zener [4] proposed the maximum growth rate criterion to establish a relation between $\sigma_{o}$ and $\sigma_{c}$, obtained by setting the first derivative of Eq. (3) equal to zero, which leads to:

$$
\sigma_{o}=2 \sigma_{c}
$$

with

$$
\sigma_{c}=\frac{2 T_{e} \sigma_{\alpha \theta}}{\rho Q\left(T_{e}-T\right)}
$$

where $T_{e}$ is the eutectoid temperature, $\sigma_{\alpha \theta}$ is the interfacial energy per unit area of the ferrite-cementite lamellar boundary in pearlite, $\rho$ is the density, $Q$ is the heat of transformation per unit mass. Combining Eqs. (4) and (5) gives an expression for $\sigma_{o}$ based on Zener's hypothesis: 


$$
\sigma_{o}=\frac{4 T_{e} \sigma_{\alpha \theta}}{\rho Q\left(T_{e}-T\right)}
$$

On the other hand, Takahashi [6] provided an empirical expression for the interlamellar spacing as a function of the temperature and the alloy content:

$$
\log \left(\sigma_{o}\right)=-2.2358+0.0986 \mathrm{Mn}-0.0543 \mathrm{Cr}+0.0337 \mathrm{Ni}-\log \left(\frac{T_{e}-T}{T_{e}}\right)
$$

where $\sigma_{o}$ is given in $\mu \mathrm{m}$ and $\mathrm{Mn}, \mathrm{Cr}$ and $\mathrm{Ni}$ in wt-\%.

Comparing Eqs. (6) and (7) the following relation should be considered:

$$
\log \left(\frac{4 \sigma_{\alpha \theta}}{\rho Q}\right)=-2.2358+0.0986 M n-0.0543 C r+0.0337 N i
$$

Substituting the alloying content of the steel in Eq. (8) and considering an approximate eutectoid temperature, $T_{e}$, of $1000 \mathrm{~K}$, Eq. (6) could be rewritten as follows:

$$
\sigma_{o}=\frac{7.1437}{(1000-T)} \text { in } \mu \mathrm{m}
$$

Figure 4 represents the variation of the interlamellar spacing as a function of undercooling calculated according to Eq. (9). Moreover, this figure shows the 
experimental values of interlamellar spacing measured using a scanning electron microscope (SEM) and a transmission electron microscope (TEM).

Table 3 and Fig. 4 show that SEM interlamellar spacing values are in good agreement with those measured using a TEM in specimens MORF1 and 2. Those values follow a tendency similar to that of the calculated curve according the model.

Contrary to TEM interlamellar spacing values, SEM values in Fig. 4 do not decrease as the degree of undercooling, $\left(T_{e}-T\right)$, increases as Mehl and co-workers [3] reported. The interlamellar spacing of the pearlite isothermally formed at $798 \mathrm{~K}$ (specimen MORF3) seems to be higher than that formed at $923 \mathrm{~K}$ (specimen MORF2) when the SEM is used in the characterisation. In addition, the SEM experimental spacing value for the specimen MORF3 is higher than its corresponding TEM value. This suggests that the experimental procedure, in particular the preparation sample method might affect the measurement of interlamellar spacing.

On the other hand, the TEM experimental value for the specimen MORF3 is in better agreement with the volume diffusion controlled growth curve than the SEM value for the same specimen. That confirms that the morphological characterisation of pearlite with an interlamellar spacing smaller than $0.085 \mu \mathrm{m}$ is almost impossible using metalographic methods based on relief contrast. Because of the difficulties associated with the sample preparation for scanning electron microscopy examination, a method based in unit cell contrast such as transmission electron microscopy is required to measure accurately the interlamellar spacing of very fine pearlite.

The SEM micrograph of specimen MORF3 in Fig. 2(c) shows an important number of pearlite colonies with broken cementite lamellae (lighter phase in the micrograph). According to Underwood's quantitative procedure [6], the interlamellar spacing is the ratio between the length of a circular grid superimposed in the 
micrograph and the number of intersections of the grid with the cementite lamellae of pearlite (Eq. (1)). If those appeared broken because of a poor preparation surface, the number of intersection would be underestimated in the quantification and the measurement of interlamellar spacing would be overestimated. Therefore, it can be concluded that the SEM experimental value for MORF3 in Fig. 4 was overestimated and the TEM experimental value is the most reliable measurement for the interlamellar spacing of specimen MORF3. As already mentioned, the TEM experimental value of that specimen confirms much better the calculations of the model.

\section{CONCLUSIONS}

1. Three different morphologies of pearlite were isothermally formed at three temperatures in a eutectoid steel (0.76C-0.91Mn-0.24Si-0.013P). It has been found that the morphological characterisation of pearlite with interlamellar spacing finer than $0.085 \mu \mathrm{m}$ using a SEM could lead to unreliable results. The strain induced during metallographic preparation of very fine pearlite destroys the lamellar morphology of pearlite and makes impossible a reliable characterisation of pearlite. In that case, it is advisable to measure the interlamellar spacing of pearlite using a TEM.

2. The proposed model to calculate the interlamellar spacing of pearlite as a function of the formation temperature has been experimentally validated at three different temperatures in the same steel. A good agreement between experimental and calculated values of the interlamellar spacings has been found in the temperature range studied. 
The authors acknowledge financial support from the European Coal and Steel Community (ECSC-7210. EC/939) and the Spanish Comisión Interministerial de Ciencia y Tecnología (CICYT-MAT95-1192-CE) 


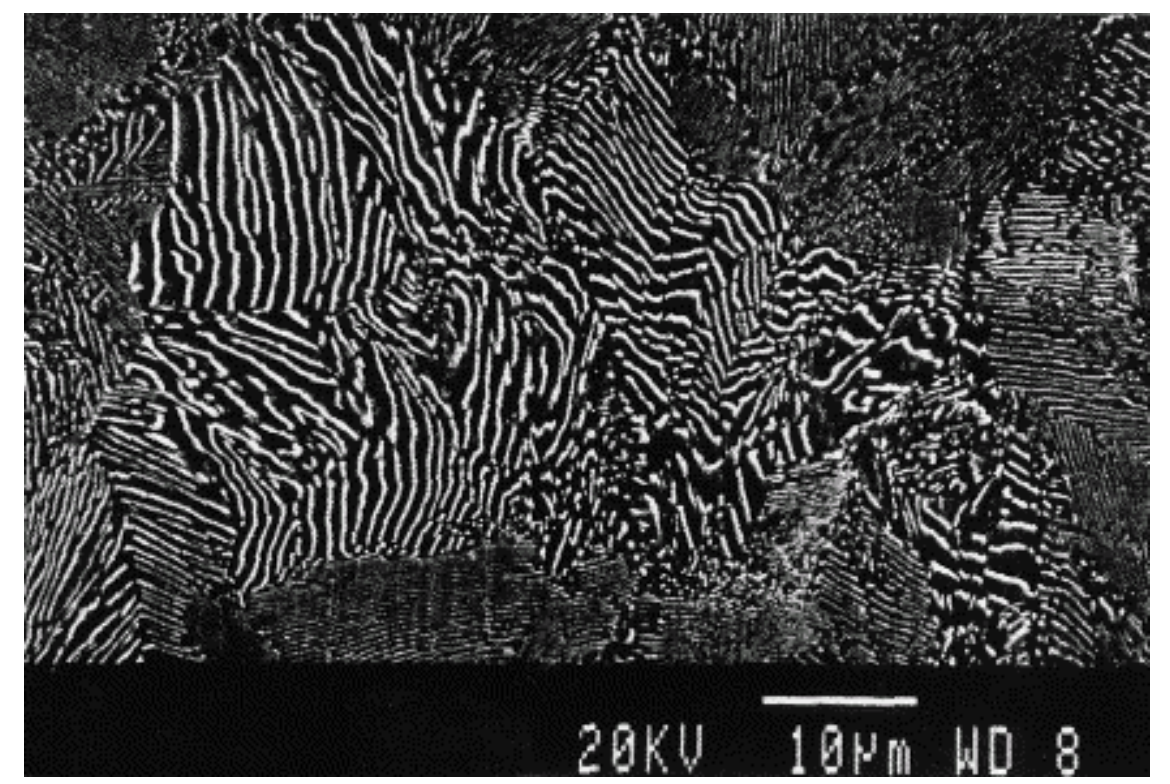

Fig. 1. Distorted lamellar structure in a pearlitic steel caused by long polishing times. 


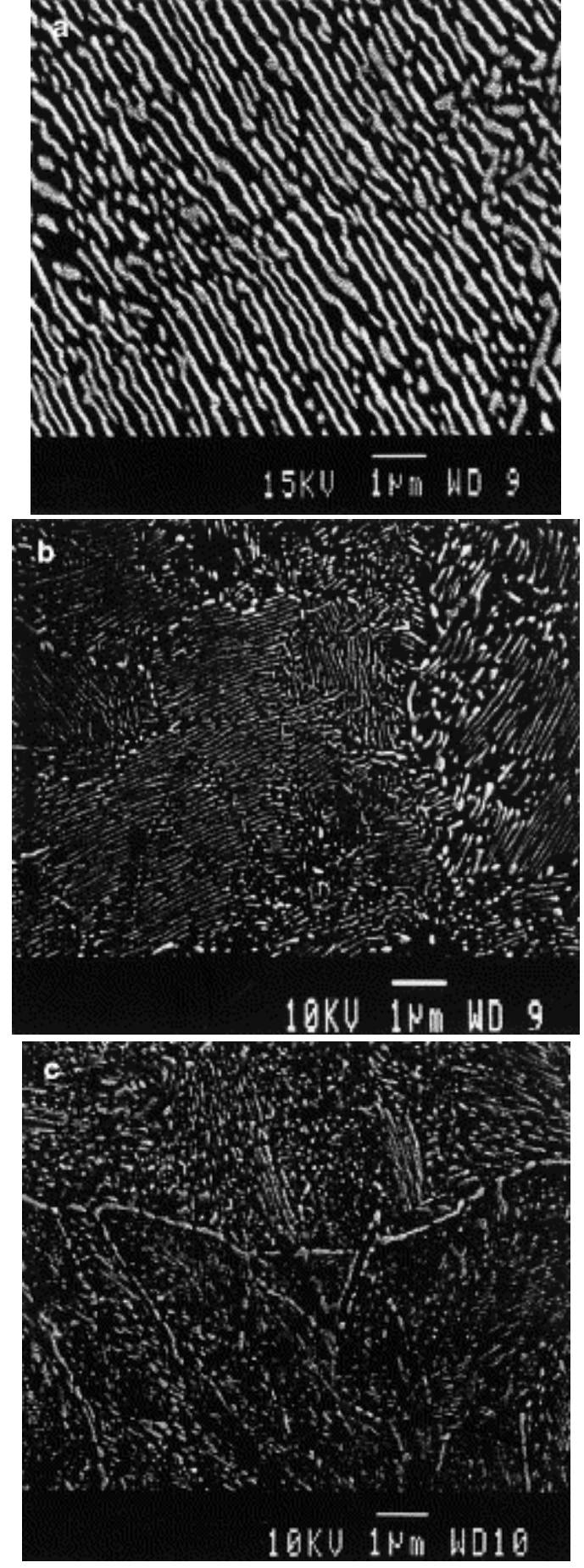

Fig. 2. SEM micrographs of (a) MORF1, (b) MORF2, and (c) MORF3 


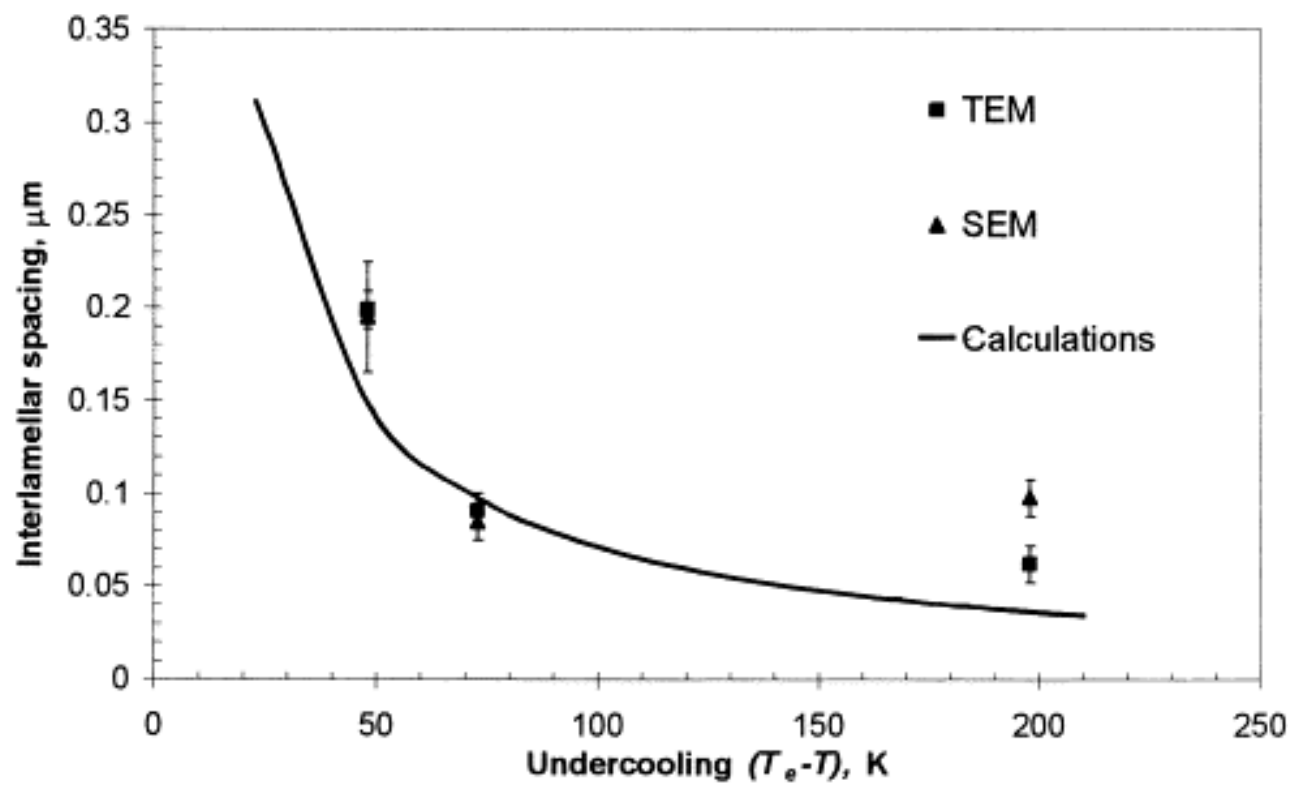

Fig. 4. Variation of interlamellar spacing as a function of undercooling. 